\title{
Efecto Morfológico de la Irradiación Láser AsGa en el Músculo Esquelético de Rata
}

\author{
Morphological Effect of AsGa Laser Irradiation on Rat Skeletal Muscle \\ "Roxana Parra Lara; **Fernando Matamala Vargas \& **Héctor Silva Mella
}

PARRA, L. R.; MATAMALA, V. F. \& SILVA, M. H. Efecto morfológico de la irradiación láser AsGa en el músculo esquelético de rata. Int. J. Morphol., 25(1):43-50, 2007.

RESUMEN: La terapia láser AsGa es ampliamente utilizada en la rehabilitación de lesiones articulares y tendinosas. Por las características físicas del haz de luz utilizado, parte de esta irradiación es absorbida por tejido muscular adyacente, por lo que se planteó medir en un modelo experimental en rata, posibles efectos morfológicos de la irradiación láser AsGa en este tejido. Se realizó un estudio morfométrico y estereológico, utilizando ratas Sprague Dawley sanas, las que fueron irradiadas, por 10 días, en la zona del vientre del músculo gastrocnemio, utilizando tres diferentes dosis 0,8 (A1 y A2), 2 (B) y 5 (C) joules/ $\mathrm{cm}^{2}$. Los miembros contralaterales no fueron irradiados (control).

Se determinó el área de la fibra muscular por recuento de puntos (morfometría) en grupo A1. La densidad de volumen de tejido conectivo se midió a través un sistema test M42 (estereología) en los grupos A2, B y C.

Los resultados obtenidos mostraron que el área de las fibras musculares fue significativamente mayor ( $\mathrm{p}=0,001)$ en los músculos irradiados que en sus controles $\left(1513 \pm 376 \mathrm{~mm}^{2}-1146 \pm 286 \mathrm{~mm}^{2}\right)$. El análisis estereológico de la densidad de volumen de tejido conectivo (Vvtc) indicó que no existieron diferencias significativas $(\mathrm{p}=0,07)$ entre músculos irradiados y controles, en ninguna de las tres dosis utilizadas. Los resultados de este estudio indican que existe, a nivel morfológico, un efecto aparentemente hipertrófico del láser AsGa en el músculo esquelético de rata.

PALABRAS CLAVE: Músculo esquelético; Láser terapia; Músculo gastrocnemio.

\section{INTRODUCCIÓN}

La emisión producida por el láser AsGa se encuentra en la gama infrarroja, en la longitud de onda de 904 nanómetros, y posee características físicas que permiten que sea utilizada en terapia física, siendo uno de los más utilizados en la llamada terapia láser de baja potencia. Los efectos beneficiosos observados clínicamente son principalmente analgésicos, antiinflamatorios y antiedematosos, bioestimulatorios y curativos (Trelles et al., 1987). Especialmente por sus efectos antiinflamatorios y analgésicos, ésta es una terapia de elección en afecciones articulares, tendinosas y musculares (Marante \& García, 1996; Gür et al., 2002). Sin embargo, al aplicar la irradiación láser en afecciones articulares o tendinosas, por ejemplo, parte de la irradiación es absorbida por tejido adyacente sano debido a las características físicas del haz de luz utilizado (Diez de los Ríos et al., 1987).
Entre los efectos observados en los tejidos biológicos, están el aumento en la producción de ATP intracelular por la estimulación mitocondrial, estímulo en la síntesis de ARN y proteínas (Greco et al., 1989; Vacca et al., 1994, 1996) y la activación de canales para el intercambio iónico relacionado con un aumento de metabolismo celular (Karu, 1989). En otros estudios morfológicos, se ha observado un efecto bioestimulatorio en el tejido fibroconectivo (Vinck et al., 2003; Shu et al., 2002 y Matamala et al., 2001). En músculos de mamíferos lesionados por diversas causas, se ha reportado un aumento de tejido funcional en procesos regenerativos (Weiss \& Oron, 1992; Houghton \& Brown, 1999; Amaral et al., 2001; Oron et al., 2001; Dourado et al., 2003). Además, se ha observado un efecto similar en músculo esquelético de anfibios (Bibikova \& Oron, 1993).

\footnotetext{
* Unidad de Anatomía Patológica, Hospital Regional Antofagasta, Antofagasta, Chile.

** Unidad de Anatomía Normal, Universidad de La Frontera, Temuco, Chile.

Patrocinio: Universidad de La Frontera, Proyecto Diufro N ${ }^{\circ} 120426$.
} 
La susceptibilidad del músculo esquelético al tratamiento estaría relacionada con su contenido mitocondrial, y la presencia de las células satélite, las que, se ha descrito, son estimuladas por la irradiación láser de baja potencia (Ben-Dov et al., 1999; Shefer et al., 2002). Es necesario recordar que esta población celular que se sitúa sobre la fibra muscular pero bajo el sarcolema, está involucrada en la regeneración del músculo esquelético, participando además en el crecimiento del músculo, aportando núcleos y la masa de su citoplasma a la fibra muscular (Goldring et al., 2002).

Con el objetivo de observar el efecto morfológico de la irradiación láser AsGa sobre el músculo esquelético normal de rata, se evaluaron en este trabajo las siguientes variables morfológicas: área de la fibra muscular y densidad de volumen de tejido conectivo (Vvtc), a través de herramientas cuantitativas morfométricas y estereológicas.

\section{MATERIAL Y MÉTODO}

Para el desarrollo de este estudio se utilizaron 20 ratas Sprague Dawley, de 11 semanas de edad, obtenidas del Bioterio de la Facultad de Medicina de la Universidad de La Frontera, Temuco, Chile, todas sanas y alimentadas con pellet ad libutum. Los equipos utilizados en este trabajo fueron proporcionados por el programa de Magister en Ciencias, mención Morfología, de la Universidad de La Frontera, Temuco, Chile.

Se dividieron las ratas en cuatro grupos de cinco (A1, A2, B y C). Se irradió el miembro posterior izquierdo de las ratas con la dosis diaria correspondiente $\left(\mathrm{A}=0,8\right.$ joules $/ \mathrm{cm}^{2}$, $\mathrm{B}=2$ joules $/ \mathrm{cm}^{2}$ y $\mathrm{C}=5$ joules $/ \mathrm{cm}^{2}$ ) durante diez días, en la zona correspondiente al vientre del músculo gastrocnemio. El láser utilizado fue de AsGa CEC Mod. IR-10E (potencia de emisión $=5 \mathrm{~mW}$; frecuencia de pulso $=5 \mathrm{kHz}$; ancho de pulso $=$ 200 nanosegundos; longitud de onda $=904 \mathrm{~nm}$ ). Los músculos gastrocnemio de la extremidad posterior derecha, no irradiados, se consideraron como controles.

Después de quince días de concluido el tratamiento se sacrificó a los animales por exceso de anestesia con éter etílico y se pesaron. Se disecaron los músculos gastrocnemio de ambos miembros, sin separar los músculos de su inserción y origen, fijándolos en formalina tamponada por 24 horas. Luego se aislaron y se pesaron por el método de Sherle (Mandarimde-Lacerda, 1995).

En el grupo A1, el espesor de los fascículos posteriores de la cabeza medial del músculo gastrocnemio, fue medido para determinar la profundidad de irradiación considerada en las me- diciones. Se tomaron cortes transversales de $1 \mathrm{~cm}$ de longitud en la zona proximal de los músculos y se procesaron histológicamente para su inclusión en Paraplast obteniendo un plano transversal del vientre muscular, en el que se realizó la determinación del área de fibras musculares en cortes histológicos de $4 \mu \mathrm{m}$ (Hematoxilina-Eosina), por recuento de puntos (Mandarim-de-Lacerda), en las zonas correspondientes a los fascículos musculares posteriores (superficiales) de la cabeza medial del músculo gastrocnemio en los miembros irradiados y no irradiados. Se evaluó la conservación del tejido conectivo.

En los grupos A2, B y C se midió el volumen de los músculos por el método de Sherle, se tomaron muestras al azar a través del método Orientator (Mattfeldt et al., 1989; Mandarim-de-Lacerda, 2003) y se procesaron histológicamente mediante un protocolo en microondas, previamente ensayado, basado en las experiencias de Boon \& Kok (1989), con objeto de mejorar la conservación del tejido conectivo. Se incluyeron en Paraplast. Se evaluó la conservación del tejido conectivo. Se midió la densidad de volumen de tejido conectivo (Vvtc), utilizándose un sistema test M42 (Mandarim-deLacerda; Mouton, 2002).

Para las mediciones y obtención de imágenes se utilizó un microscopio óptico Olympus CX31 conectado a un monitor Sony 21" Fv300 mediante cámara invertida Moticam 480-digital análoga.

Las mediciones fueron sometidas a un análisis de $t$ de student en el programa computacional Stata 7.0. Se consideró un nivel de significancia de $5 \%(\mathrm{p}<0,05)$.

\section{RESULTADOS}

Observación macroscópica. Los músculos gastrocnemio de rata Sprague Dawley se observaron constituidos por una cabeza lateral y una cabeza medial, de mayor volumen, y subdividida en cuatro fascículos, superficiales y profundos. Estos fascículos se encontraron rodeados de fascia. La distancia entre la fascia superficial y el límite de los fascículos superficiales fue de $3,5 \mathrm{~mm}$.

Observación histológica: Se observaron fibras musculares bien constituidas con núcleos de ubicación excéntrica. En el grupo A1 el tejido conectivo presentó una conservación regular, mientras que en el grupo A2 se conservó de manera óptima (Fig. 1). La fascia limitante de los fascículos musculares estaba constituida de tejido conectivo denso. No se encontraron evidencias de fibrosis cicatrizal, inflamación o necrosis. Asimismo, no se observaron fibras musculares de núcleo central (fibras musculares jóvenes) (Figs. 1a, 1b). 


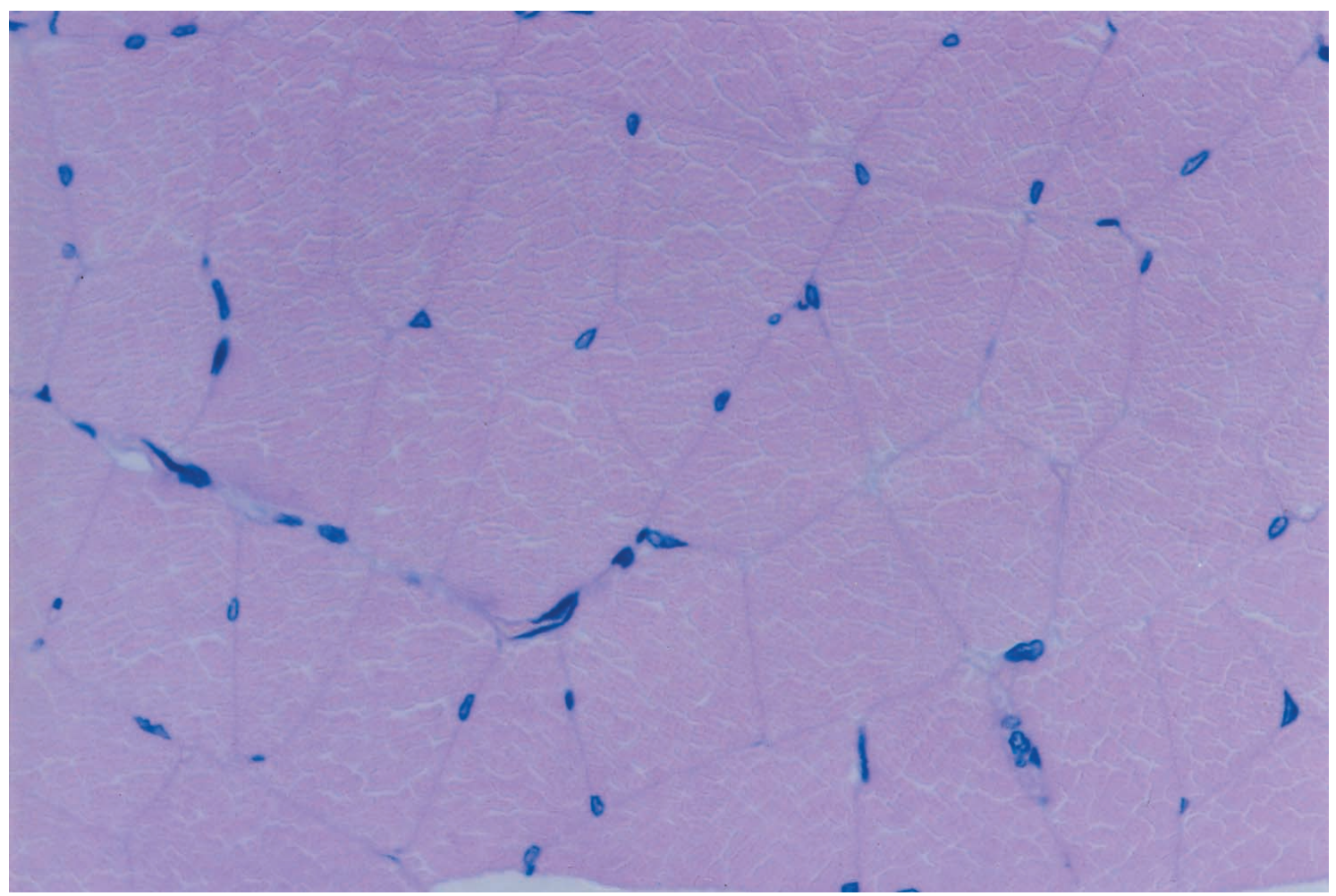

Fig. 1a. Músculo gastrocnemio procesado en microondas. Hematoxilina-Eosina. 400 x.

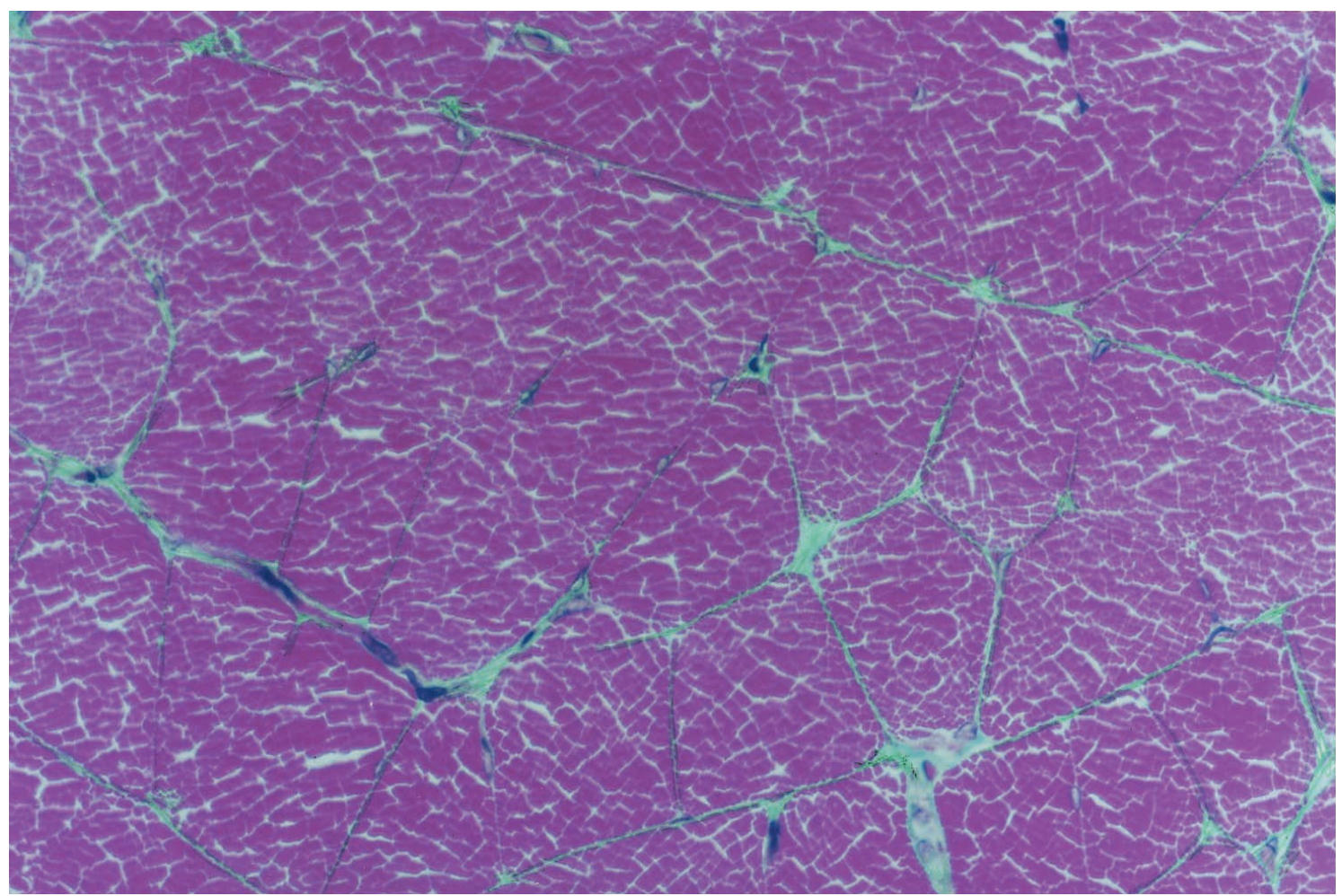

Fig. 1b. Músculo gastrocnemio procesado en microondas. Tricrómico de Masson. 400 x. 
Área de las fibras irradiadas y control, grupo A1 (0,8 joule $/ \mathbf{c m}^{2}$ ): Considerando el total de observaciones en músculos controles e irradiados, tanto el área promedio, el área mínima y el área máxima obtenida, fueron mayores en los músculos irradiados. La D.E. fue relativamente mayor en el grupo de fibras musculares irradiadas (Tabla I). En la Tabla II se detallan los promedios y D.E. por rata.
El resultado de este análisis mostró que tomando en cuenta el total de observaciones ( $\mathrm{nI}=500 ; \mathrm{nC}=500)$, el área de la fibra muscular es significativamente mayor en los músculos irradiados que en los controles. Los patrones de diferencias en todos los sujetos fueron altamente significati$\operatorname{vos}(\mathrm{p}<0.001)$.

Tabla I. Promedio del área $\left(\mathrm{mm}^{2}\right)$ y desviación estándar de las fibras musculares irradiadas con láser AsGa $(\mathrm{n}=500)$ y controles Grupo A1 ( $\mathrm{n}=500)$ en músculo gastrocnemio de ratas Sprague Dawley obtenidas del Bioterio de la Facultad de Medicina de la Universidad de La Frontera, Temuco, Chile.

\begin{tabular}{|c|c|c|c|c|}
\hline Variable & Promedio área $\left(\mathrm{mm}^{2}\right)$ & Desviación estándar & Mínimo & Máximo \\
\hline Fibras musculares irradiadas & 1513 & 376 & 600 & 2800 \\
\hline Fibras musculares control & 1146 & 286 & 400 & 2200 \\
\hline
\end{tabular}

Tabla II. Promedio del área $\left(\mathrm{mm}^{2}\right)$ y desviación estándar del área de las fibras musculares irradiadas con láser AsGa $(n=100)$ y controles Grupo A1 $(n=100)$ en músculo gastrocnemio de ratas Sprague Dawley obtenidas del Bioterio de la Facultad de Medicina de la Universidad de La Frontera, Temuco, Chile.

\begin{tabular}{ccccc}
\hline & \multicolumn{2}{c}{ Fibras musculares irradiadas } & \multicolumn{2}{c}{ Fibras musculares control } \\
Rata & Promedio & Desviación estándar & Promedio & Desviación estándar \\
\hline 1 & 1536 & 413 & 1172 & 316 \\
2 & 1675 & 375 & 1112 & 199 \\
3 & 1561 & 366 & 1320 & 359 \\
4 & 1477 & 349 & 1094 & 199 \\
\hline
\end{tabular}

Densidad relativa de tejido conectivo (Vvtc\%) en músculos gastrocnemio irradiados y control. Muestreo aleatorio. El análisis de los datos obtenidos del estudio estereológico indica, con un $95 \%$ de confianza que no hay diferencias significativas en el Vvtc, entre músculos gastrocnemio irradiados y control $(\mathrm{p}=0,070)$, ni entre las dosis estudiadas en relación con el contenido de tejido conectivo (p=0,072) (Fig. 2).

Se calculó el promedio de los promedios obtenidos, de tal manera de obtener el porcentaje aproximado de tejido conectivo de un músculo gastrocnemio de rata Sprague Dawley de 11 semanas de edad, este resultó 6,75\%. El volumen promedio de los músculos gastrocnemio fue de 1,108 $\mathrm{ml}$, lo que permite inferir un contenido promedio de 0,122 $\mathrm{ml}$ de tejido conectivo.

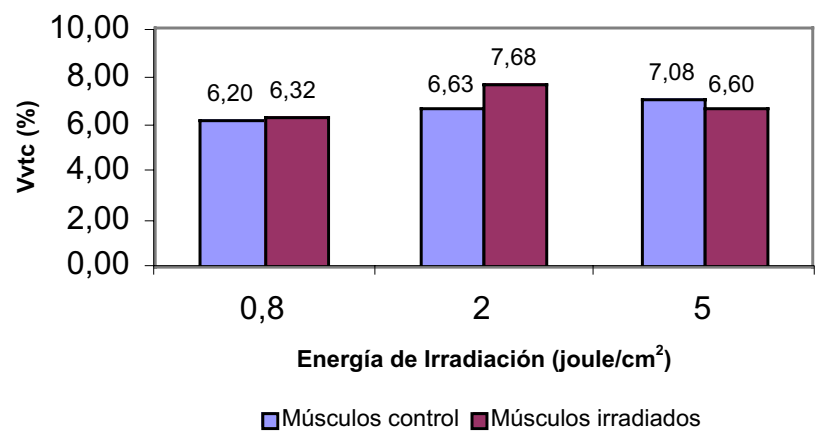

Fig. 2. Distribución del Vvtc en los músculos gastrocnemio de rata Sprague Dawley control e irradiados con láser AsGs contres diferentes dosis. $\mathrm{J}=$ joule. Grupo A2 $\left(0,8 \mathrm{~J} / \mathrm{cm}^{2}\right), \mathrm{B}\left(2 \mathrm{~J} / \mathrm{cm}^{2}\right)$ y $\mathrm{C}(5 \mathrm{~J} /$ $\left.\mathrm{cm}^{2}\right)$. 


\section{DISCUSIÓN}

De acuerdo a las características de la radiación láser de baja potencia, se puede inferir que la radiación dirigida hacia un punto de interés terapéutico, es también recibida por tejidos adyacentes sanos.

Experiencias en cultivo con aislados mitocondriales de rata, irradiados con láser de baja potencia $\left(5\right.$ joules $\left./ \mathrm{cm}^{2}\right)$, se observó un aumento de productos de transcripción de ARN, medido por aumento de incorporación de un producto radioactivo (a-[32P] UTP), obtenido en relación a cultivos control no irradiados (Greco et al.). Vacca et al. (1994) observaron que al irradiar templados de ADN, junto con enzimas de transcripción y traducción por separado en cultivo ( 2 joules/ $\mathrm{cm}^{2}$ ), aumentó tanto la producción de ARN, como la producción de proteínas. Se comprobó también que el aumento de síntesis proteica ocurre tanto a nivel mitocondrial como citosólico (Vacca et al., 1996).

En el músculo esquelético se han demostrado efectos morfológicos atribuibles a la estimulación de los procesos, biosintéticos de la célula, en la regeneración de tejido muscular esquelético lesionado. En músculos gastrocnemio de rata seccionados parcialmente, se observó un porcentaje significativamente mayor de fibras musculares en la zona de la lesión en músculos irradiados con láser He-Ne, comparados con músculos controles no irradiados $(13,9 \pm 1 \%$ y $7,8 \pm 1 \%)$ (Weiss \& Oron). Por su parte, Houghton \& Brown, reportaron que la administración de dosis diarias de 0,23 y 1,37 joules/ $\mathrm{cm}^{2}$ de láser AsGa en miembros lesionados de ratón mantenidos en cultivo, disminuyen significativamente el área de la lesión. Sin embargo, la administración de dosis diarias de 4,58 joules $/ \mathrm{cm}^{2}$, produjo un detrimento en la morfología de la lesión. Amaral et al., utilizando dosis de 2,6; 8,4 y 25 juoles/ $\mathrm{cm}^{2}$ en músculo tibial anterior de ratón lesionado con miotoxina ACL, observaron que sólo con 2,6 joules $/ \mathrm{cm}^{2}$ se presentaron cambios en las fibras musculares como aumento de la densidad mitocondrial y del promedio del área de la fibra muscular irradiada en comparación con el control, sugiriendo que sólo dosis bajas tendrían un efecto benéfico en la reparación tisular. Dourado et al., indican que en músculos de ratón expuestos al veneno de la serpiente Bothrops mojen, una significativa disminución de la necrosis muscular en músculos tratados con dosis diarias de 4 joules $/ \mathrm{cm}^{2}$ de láser AsGa. Sin embargo, no encontraron en la literatura reportes acerca de los efectos de esta terapia en músculo esquelético sano en relación a posibles efectos morfológicos no deseados.

Los resultados obtenidos en este estudio indican que en el primer grupo de ratas (grupo A1) Sprague Dawley el área de las fibras del músculo gastrocnemio aumentó en los músculos irradiados con láser AsGa 0,8 joules $/ \mathrm{cm}^{2}$ en los fascículos más próximos a la irradiación ( $\mathrm{p}<0,001)$, siendo el promedio de las áreas $1513 \pm 376 \mathrm{~mm}^{2}$ en músculos irradiados versus $1146 \pm 286 \mathrm{~mm}^{2}$ en músculos controles. El espesor máximo de estos fascículos se midió en todos los músculos, encontrando que la distancia promedio entre la superficie del músculo y la fascia limitante de los fascículos posteriores en la línea mediana de la cabeza medial del músculo gastrocnemio, fue de 3,5 mm, en músculos irradados y controles.

Amaral et al., que emplean el láser HeNe en tejidos musculares lesionados de ratón, explican que sólo un $25 \%$ de la emisión de un láser HeNe, traspasa efectivamente la piel y llega al músculo. Si se aplican los cálculos de Diez de los Ríos et al., un $45 \%$ de la potencia de salida del láser habría sido absorbida a los 3,5 mm, en caso de que no hubiese existido la piel, pero esto se disminuye a un cuarto según lo observado por Amaral y su grupo. La emisión que efectivamente habría llegado al límite de los fascículos posteriores de la cabeza medial de los músculos gastrocnemio, sería de 0,09 joules/ $\mathrm{cm}^{2}$, aproximadamente. Lo que significa que la mayor parte de la irradiación habría sido ya absorbida a esta profundidad.

Una posible explicación a la estimulación observada es la participación de las células satélite. Las células satélite son células con capacidad miogénica, que están relacionadas íntimamente con la fibra muscular, bajo la lámina basal o sarcolema. Esta población celular normalmente no presenta actividad mitótica en el músculo adulto, pero se activa o prolifera en el proceso de regeneración del músculo esquelético. Esta población celular participaría no sólo en la regeneración del músculo esquelético, sino en el crecimiento de éste, aportando núcleos y la masa de su citoplasma a la fibra muscular (Goldring et al.). Lo anterior sería una posible explicación al efecto observado, ya que las células satélite son estimuladas por la irradiación láser de baja potencia, activando su ciclo celular e incorporación a fibras musculares en cultivo (BenDov et al.; Shefer et al.).

En la observación histológica de los músculos irradiados no se encontraron zonas de fibrosis ni de necrosis. Además, no se observaron fibras musculares jóvenes, las que se identifican por la ubicación central de sus núcleos (Fig. 1).

La aparición de miofibras jóvenes refleja actividad regenerativa del tejido, y se puede inferir, por su ausencia, que no existió daño tisular reciente. En este sentido se debe recordar que la toma de muestra se realizó quince días posttratamiento, período en el cual ya se observaría la reacción del tejido al posible daño tisular provocado. El efecto observado correspondería a una estimulación metabólica del tejido, con un aumento del área de las fibras musculares, al menos, en la zona de irradiación. 
En las fibras musculares irradiadas con 0,8 joules $/ \mathrm{cm}^{2}$, se observó una anisocitosis leve, es decir, no se observó una arquitectura tisular uniforme, a diferencia de los músculos control, situación que se corroboró con el análisis estadístico que mostró que la D.E. de las áreas de las fibras de músculos irradiados fue mayor que en las fibras de músculos control. Esto podría deberse a que la absorción de la irradiación láser disminuiría con la profundidad, resultando en una respuesta no homogénea en el tejido.

Respecto al efecto del láser en el tejido conectivo, variados autores destacan la influencia de la irradiación láser en el tejido conectivo Matamala et al., reportaron un aumento del perineuro de nervios isquiáticos de conejo, de aproximadamente un $25 \%$ ( $21 \mathrm{~mm}$ en nervios controles y $27,8 \mathrm{~mm}$ en nervios irradiados). Por otro lado, cultivos de fibroblastos obtenidos de embriones de pollo, se trataron con láser AsGaAl $(830 \mathrm{~nm})$, se observó una mayor proliferación de fibroblastos en las monocapas celulares irradiadas en relación con las monocapas celulares no irradiadas (Vinck et al). Sin embargo, al utilizar dosis altas el proceso de revertiría, disminuyendo considerablemente la proliferación celular (Shu et al.).

No se obtuvieron datos del contenido de tejido conectivo, en la primera experiencia con el grupo A1 (muestreo de fascículos musculares adyacentes al punto de irradiación; irradiados con 0,8 joules $/ \mathrm{cm}^{2}$ por diez días), ya que la conservación del peri y endomisio no fue óptima. Sin embargo, luego de estudiar los músculos gastrocnemio del grupo A2 como un todo, a través de estereología, los resultados no mostraron diferencias significativas en la densidad de volumen tejido conjuntivo (Vvtc) en los músculos analizados. El grupo A2 tuvo el mismo protocolo de irradiación pero una mejora en la recuperación del tejido conectivo. La herramienta utilizada (Orientator) no permitió muestrear la zona de irradiación, ya que se muestrea el músculo completo de manera aleatoria. Además, en los grupos B y C (diez sesiones de 2 y 5 joules/ $\mathrm{cm}^{2}$, respectivamente), tampoco se encontraron diferencias significativas en la densidad de volumen de tejido conectivo entre músculos irradiados y control. Sin embargo, estos resultados no descartan, un efecto local en el tejido conectivo.

No obstante, se pueden aportar algunos datos a la morfología del músculo gastrocnemio de rata Sprague Dawley. Se calculó un promedio de los promedios de Vvtc obtenidos en músculos control (Vvtc= 6,75\%). Esto quiere decir que en $1 \mathrm{ml}$ de músculo gastrocnemio de rata Sprague Dawley de 11 semanas de edad, hay teóricamente en promedio $0,675 \mathrm{ml}$ de tejido conjuntivo. El promedio de volumen de los músculos fue de $1,108 \mathrm{ml}$, por lo que es posible asegurar que para este volumen de músculo, habría, en promedio, $0,122 \mathrm{ml}$ de tejido conjuntivo. No hubo una relación entre el peso de las ratas y el volumen de los respectivos músculos gastrocnemio.

El estudio pretendió estudiar sólo desde el punto de vista morfológico, los posibles efectos de la terapia AsGa en el tejido muscular esquelético. No se encontraron evidencias de fibrosis, necrosis, inflamación o fibras musculares jóvenes, habiéndose descartado daño tisular reciente. El aumento del área de las fibras musculares observado en las fibras irradiadas fue compatible con un efecto hipertrófico. Se descartó un efecto hiperplásico por la ausencia de miofibrillas jóvenes, considerando que las muestras se tomaron sólo quince días terminado el tratamiento. Siendo éste un estudio de corte transversal queda planteada la interrogante acerca de la duración de este efecto, considerando que el músculo esquelético es un órgano de alta plasticidad.

AGRADECIMIENTOS.Agradecemos al Programa Magister en Ciencias mención Morfología de la Universidad de La Frontera, Temuco, Chile, por haber proporcionado la mayoría de los equipos, reactivos e insumos utilizados en este trabajo. Al profesor Juan José Orellana por la orientación y apoyo en los análisis estadísticos.

PARRA, L. R.; MATAMALA, V. F. \& SILVA, M. H. Morphological effect of AsGa laser irradiation on rat skeletal muscle. Int. J. Morphol., 25(1):43$50,2007$.

SUMMARY: Whether laser therapy is widely used in healing therapies of injured muscle, there are no reports about its morphological effects on healthy muscle. A morphometrical and stereological study was performed with healthy Sprague Dawley rats, all irradiated with AsGa laser directly on the skin of the gastrocnemius muscle belly area for ten days, using three different daily doses on groups of five rats 0,8 (A1 y A2), 2 (B) y 5 (C) joules/ $\mathrm{cm}^{2}$ on the left posterior limb. The right limb was no irradiated, so it was used as control.

The muscle fiber area was determinate by point counting (morphometry) on group A1. Density of connective tissue contain (Vvtc) was determinate by M42 test (stereology) on A2, B and C group.

The analysis of all the 500 fiber areas obtained from A1 group muscles and 500 fiber areas from its control muscles show that the mean fiber area was $1513 \pm 376 \mathrm{~mm}^{2}$ for laser treated gastrocnemius muscles and $1146 \pm 286 \mathrm{~mm}^{2}$ for non irradiated control gastrocnemius muscles. Regression analysis showed that the fiber area was higher on treated muscles $(\mathrm{p}<0,001)$. Stereological analysis of Vvtc results showed no significance difference between treated and control muscles in none of the used doses $(\mathrm{p}=0.07)$.

AsGa laser therapy produce morphological effects on healthy muscle.

KEY WORDS: Skeletal muscle; Laser therapy; Gastrocnemius muscle. 


\section{REFERENCIAS BIBLIOGRÁFICAS}

Amaral, A. C.; Parizotto, N. A. \& Salvini T. F. Dosedependency of low-energy HeNe laser effect in regeneration of skeletal muscle in mice. Lasers Med. Surg., 16:44-51, 2001.

Ben-Dov, N.; Shefer, G.; Irinitchev, A.; Wernig, A.; Oron, U. \& Hevely, O. Low-energy laser irradiation affects satellite cell proliferation and differentiation in vitro. Biochem. Biophys. Acta, 1448:372-80, 1999.

Bibikova, A. \& Oron, U. Promotion of muscle regeneration in the toad (Bufo viridis) gastrocnemius muscle by lowenergy laser irradiation. Anat. Rec., 235:374-80, 1993.

Boon, M. \& Kok, L. Microwave cookbook of pathology. The art of microscopic visualization. $2^{\text {nd }}$ ed. Coulomb press Leyden, Leiden, Netherlands, 1989.

Diez de los Rios, A.; Sendra, F.; Pastor, M.; Labajos, M. \& Martinez, M. Estudio de la absorción de la radiación láser de He-Ne y As-Ga por tejidos biológicos. Inv. Clin. Laser, 3:98-100, 1987.

Dourado, D.; Fávero, S.; Baranauskas, V. \& Cruz-Höfling, M. A. Effects of the Ga-As irradiation on myonecrosis causes by Bothrops mojen snake venom. Lasers Surg. Med., 33:352-7, 2003.

Goldring, K.; Partridge, T \& Watt, D. Muscle stem cells. J. Pathol., 197(4):457-67, 2002.

Greco, M.; Guisa, G; Perlino, E.; Marra, E. \& Quagliariello, E. Increase in Rna and protein synthesis by mitochondria irradiated with helium-neon laser. Biochem. Biophys. Res. Comm., 163(3):1428-34, 1989.

Gür, A.; Karakoc, M.; Nas, K.; Cevik, R.; Sarac, J. \& Demir, E. Efficacy of low power laser therapy in fibromyalgia: a single-blind, placebo-controlled trial. Lasers. Med. Sci., 17:57-61, 2002.

Houghton, P.E. \& Brown, J.L. Effect of low level laser on healing in wounded fetal mouse limbs. Laser Therapy, 11(2):54-68, 1999

Karu, T. L. Photobiology of low-power laser effects. Health Physiol., 56:671-704, 1989.

Mandarim-de-Lacerda, C. A. Métodos cuantitativos em morfología. Eduerj, Universidade do Rio de Janeiro, Brasil, 1995.
Mandarim-de-Lacerda, C. A. Stereological tools in biomedical research. An. Acad. Bras. Cienc., 75(4):469-86, 2003.

Marante, M. I. \& García, J. A. Lásers de baja potencia: resultados de su aplicación en la rehabilitación. Rev. Cubana Ortop. Traumatol., 10(1):51-7, 1996

Matamala, F.; Veuthey, C.; Molina, B.; Silva, H. \& Henriquez, J. Efecto del láser infrarrojo de uso clínico sobre el perineuro de nervio isquiático de conejo. Arch. Med. Vet., 33(2):253-9, 2001.

Mattfeldt, T.; Mall, G.; Von Herbay, A. \& Möller, P. Stereological investigation of anisotropic structures with orientator. Acta stereologica, 8(2):671-6, 1989.

Mouton, P. Principles and practices of unbiased stereology. The Jhons Hopkins University Press, Baltimore, 2002.

Oron, U.; Kaakobi, T.; Oron, A.; Mordechovitz, D.; Shofti, R.; Hayam, G.; Dror, U.; Gepstein, L.; Wolf, T.; Haudenschild, C. \& Ben Haim, Sh. Low-energy laser irradiation reduces formation of scar tissue after miocardial infarction in rats and dogs. Circulation, 103(2):296-303, 2001.

Shefer, G.; Partridge, T.; Heslop, L.; Gross, J.; Oron, U. \& Halevy, O. Low-energy laser irradiation promotes the survival and cell cycle entry of skeletal muscle satellite cells. J. Cell science, 115(7):1461-9, 2002.

Shu, B.; Wuzongyao, L.; Zeng, D.; Feng, G. \& Lin, Y. Experimental study on $\mathrm{He}-\mathrm{Ne}$ irradiation to inhibit scar fibroblast growth in culture. Chin. J. Traumatol., 5(4):246-9, 2002.

Trelles, M. A.; Mayayo, E.; Mester, A. \& Rigau, J. Low power laser therapy. Experimental and clinical data with special reference to Spain. SJA\&ET, 2:80-100, 1987.

Vacca, R.; Marra, E.; Quagliariello, E. \& Greco, M. Increase of both transcription and translation activities following separate irradiation of the in vitro system components with He-Ne laser. Biochem. Biophys. Res. Commun., 203(2):991-7, 1994.

Vacca, R.; Marra, E.; Passarella, S.; Petragallo, V. \& Greco, M. Increase in cytosolic and mitochondrial protein synthesis in rat hepatocytes irradiated in vitro by $\mathrm{He}-\mathrm{Ne}$ laser. J. Photochem. Photobiol., 34:197-202, 1996. 
Vinck, E. M.; Cagnie, B. J.; Cornelissen, M. J.; Declereq, H.A. \& Cambier, D. C. Increased fibroblast proliferation induced by ligth emitting diode and low power laser irradiation. Lasers Med. Sci., 18:95-9, 2003.

Weiss, N. \& Oron, U. Enhancement of muscle regeneration in the rat gastrocnemius muscle by low energy laser irradiation. Anat. Embryol., 186:497-503, 1992.
Dirección para correspondencia

T. M. Roxana Parra Lara, M.Sc. Unidad de Anatomía Patológica Hospital Regional Antofagasta

Avda. Argentina 1962

Antofagasta

CHILE

Email:chanitaparra@yahoo.com

Recibido : 07-09-2006

Aprobado: 22-11-2006 